\title{
Analysis of stacked rotated gratings
}

Gregory P. Nordin

nordin@byu.edu

P. C. Deguzman

J. Jiang

Follow this and additional works at: https://scholarsarchive.byu.edu/facpub

Part of the Electrical and Computer Engineering Commons

\section{Original Publication Citation}

J. Jiang, P. C. Deguzman, and G. P. Nordin, "Analysis of stacked rotated gratings," accepted for publication in Applied Optics (27)

\section{BYU ScholarsArchive Citation}

Nordin, Gregory P.; Deguzman, P. C.; and Jiang, J., "Analysis of stacked rotated gratings" (2007). Faculty Publications. 933.

https://scholarsarchive.byu.edu/facpub/933

This Peer-Reviewed Article is brought to you for free and open access by BYU ScholarsArchive. It has been accepted for inclusion in Faculty Publications by an authorized administrator of BYU ScholarsArchive. For more information, please contact ellen_amatangelo@byu.edu. 


\title{
Analysis of stacked rotated gratings
}

\author{
Jianhua Jiang, Panfilo C. Deguzman, and Gregory P. Nordin
}

\begin{abstract}
We study the rigorous design and analysis of stacked rotated gratings (SRGs) for novel photonic devices. A concept of sampling frequency is proposed to systematically design SRGs. With the help of a rectangular sampling lattice, we extend the standard 3D rigorous coupled-wave analysis (RCWA) algorithm to the analysis of SRGs. The resultant SRG-RCWA algorithm can be used to exactly analyze SRGs that have grating parameters that match the Fourier expansion represented by the sampling lattice. SRGs that do not fall in this category can often still be closely approximated with our approach. The SRG-RCWA algorithm has been successfully applied to the characterization of two fabricated SRGs that are designed to function as circular polarization filters for an IR imaging polarimetry system. The agreement between numerical SRG-RCWA results and experimental measurements demonstrates its validity and usefulness. (C) 2007 Optical Society of America
\end{abstract}

OCIS codes: $\quad 050.1950,050.1960,230.5440,260.5430$.

\section{Introduction}

Gratings have extensive applications in many optical systems. ${ }^{1}$ Thus it is not surprising that stacked or cascaded multilayer gratings (SMGs) also have numerous applications. ${ }^{2-4}$ Usually all of the gratings in a SMG are $1 \mathrm{D}$ or $2 \mathrm{D}$ gratings that have the same grating period(s) and angular orientation. This greatly limits the functionality and design freedom of SMG-based devices. A more general structure of SMGs is the stacked rotated grating (SRG), in which the gratings may have different grating periods and may be rotated with respect to each other. In this paper we focus on the study of SRGs composed of rotated $1 \mathrm{D}$ gratings.

The additional freedom of different grating periods and rotational orientations for each grating layer enables SRGs to realize device functions that are not achievable with conventional SMGs. However, little

\footnotetext{
When this research was performed J. Jiang (jjiang@breault.com) was with the Nano and Micro Devices Center, the University of Alabama in Huntsville, Huntsville, Alabama 35899, USA. He is now with the Breault Research Organization, Inc., 6400 East Grant Road, Suite 350, Tucson, Arizona 85715, USA. P. C. Deguzman is with the Center for Optoelectronics and Optical Communications, University of North Carolina at Charlotte, Charlotte, North Carolina 28223, USA. G. P. Nordin is with the Department of Electrical and Computer Engineering, Brigham Young University, Provo, Utah 84602, USA.

Received 1 March 2006; revised 1 November 2006; accepted 2 November 2006; posted 7 November 2006 (Doc. ID 68402); published 20 February 2007.

0003-6935/07/081177-07\$15.00/0

(C) 2007 Optical Society of America
}

attention has been paid to SRGs in the literature. Hwang and Peng 5 designed a SRG structure with two gratings (bigrating) as a beam splitter. Lin and Fleming ${ }^{6}$ constructed a SRG with orthogonal 1D gratings as a woodpile type 3D photonic crystal. More recently Deguzman and Nordin ${ }^{7}$ developed an integrated SRG as a circular polarization filter for IR imaging polarimetry applications. In Deguzman's device, a subwavelength metallic grating is used as a wire-grid polarizer and another dielectric subwavelength grating is designed to function as a formbirefringent quarter-wave plate, which is rotated $45^{\circ}$ with respect to the wire-grid polarizer.

A key reason for lack of attention to SRGs is likely the absence of theoretical tools to analyze and design SRGs. As illustrated in Fig. 1, the diffraction of SRGs is generally a $3 \mathrm{D}$ problem because of the different orientations of the grating layers in the structure. The problem is further complicated by their different grating periods. As a result, the number of diffracted orders generated by SRGs may increase exponentially with the number of gratings, and their wave vectors form an irregular lattice in $k$ space. This irregular $k$-space lattice makes the standard 3D rigorous grating models for SMGs with $2 \mathrm{D}$ gratings, such as the coupled-wave analysis method ${ }^{8,9}$ (3D RCWA), inapplicable to the analysis of SRGs. In standard 3D RCWA, it is required that all of the gratings must have the same periods and perfect alignment. This requirement implies that the diffracted orders form a regular rectangular lattice in $k$ space. Therefore the permittivity functions of the $2 \mathrm{D}$ gratings can be expanded into a Fourier series with the same fundamental frequencies. 


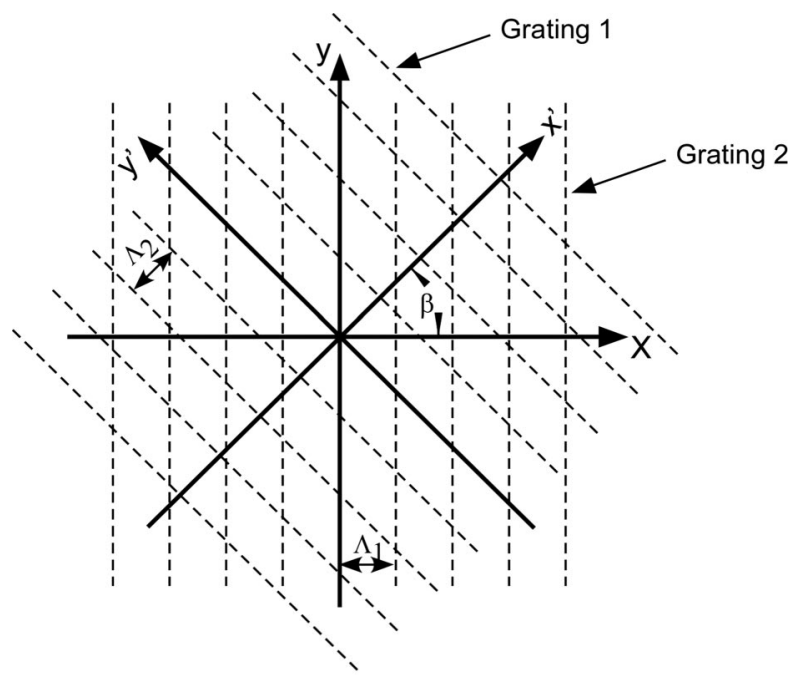

(a) Top View

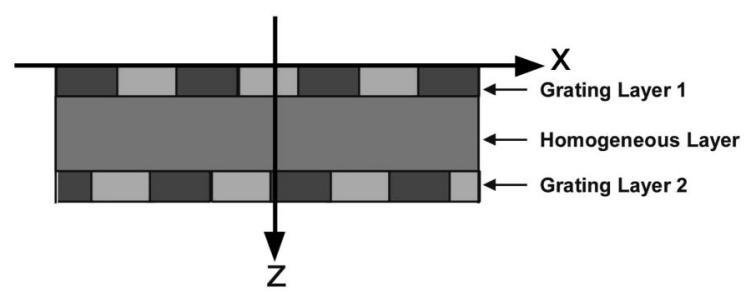

(b) Side View

Fig. 1. Schematic of the stacked rotated grating structure.

Currently there exist no rigorous diffraction models for general SRGs. For a simple bigrating structure as proposed in Ref. 5 and shown in Fig. 1, Hwang and Peng 5 developed a diffraction model based on multiple reflections of different diffracted orders of the constituent gratings. In Hwang and Peng's model, one of the grating vectors in the structure is projected onto the other grating vector such that a bigrating is a structure that is doubly periodic in one direction and singly periodic in the other direction. Therefore their model can be simply understood as an extension of the rigorous diffraction model for conical mounting for the treatment of doubly periodic structures. Theoretically it is possible to further extend Hwang and Peng's model to the analysis of general multiple-layer SRGs. Numerically, however, it is not feasible because of the exponential increase in the number of diffraction orders generated by the gratings in SRGs so that the size of the resultant matrices is too large to fit into the computer memory.

In this paper we introduce a sampling frequency concept such that the standard RCWA method can be extended to the analysis and design of some types of multilayer SRGs. We call the modified 3D RCWA method the stacked rotated grating RCWA (SRGRCWA). In SRG-RCWA, a rectangular sampling $k$ lattice with unit sampling constants $f_{x}$ and $f_{y}$ is sought such that all of the diffraction orders of the gratings fit onto the sampling lattice grid. If such a sampling lattice exists, the permittivity functions of the gratings in the SRGs can be simultaneously expanded into a Fourier series with fundamental frequencies of $f_{x}$ and $f_{y}$. Therefore the standard RCWA algorithms can be applied. This sampling frequency concept is particularly useful and flexible when designing SRGs because the sampling frequencies can be chosen freely and independently. In conjunction with the sampling numbers, the corresponding grating period and orientation can be easily determined, as explained below.

The rest of the paper is organized as follows. In Section 2, we first introduce the sampling frequency concept for SRG-RCWA and then use numerical results for a simple SRG that can be analyzed by standard 3D RCWA to validate SRG-RCWA. Section 3 presents a detailed analysis using the SRG-RCWA of two fabricated integrated circular polarization filters composed of SRGs. In both cases the SRG-RCWA results match measurements very well. Comments and conclusions are presented in Section 4.

\section{Basic Concept and Implementation of SRG-RCWA}

\section{A. Sampling Frequency Concept for SRG-RCWA}

We illustrate the sampling frequency concept of SRGRCWA in Fig. 2. To design a multilayer SRG, we first draw a rectangular lattice in $k$ space with arbitrary unit sampling constants $f_{s x}$ and $f_{s y}$ and assume that all the diffraction orders of the SRG can locate only on the grid points of the lattice. Now it is interesting to see that every vector that starts from the coordinate origin and ends at a lattice grid point stands for a possible grating vector for a 1D grating layer in the SRG. We further introduce the concept of integer sampling numbers $\left(n_{x}, n_{y}\right)$, which are the coordinates for every grid point in the lattice. Then it is very easy to calculate the grating parameters of the grating

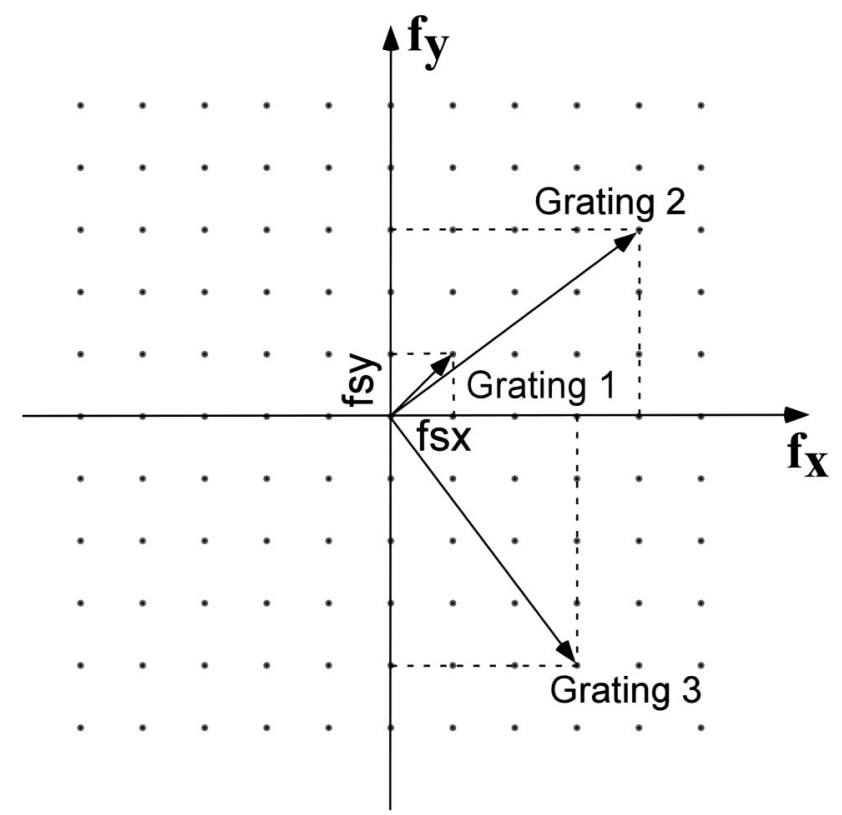

Fig. 2. Frequency sampling lattice of SRG-RCWA and three sample grating $\bar{K}$-vectors. 
associated with every lattice point. With a simple geometric relation, the grating period $\Lambda$ and the angle $\beta$ between the grating vector and the $f_{x}$ axis can be calculated as

$$
\begin{aligned}
\Lambda & =\frac{1}{\left[\left(n_{x} f_{s x}\right)^{2}+\left(n_{y} f_{s y}\right)^{2}\right]^{1 / 2}}, \\
\sin \beta & =\frac{n_{y} f_{s y}}{\left[\left(n_{x} f_{s x}\right)^{2}+\left(n_{y} f_{s y}\right)^{2}\right]^{1 / 2}}=n_{y} f_{s y} \Lambda .
\end{aligned}
$$

For instance, in Fig. 2, if $f_{s x}=f_{s y}=1 \mu \mathrm{m}^{-1}$, then the parameters of the three illustrated gratings are

Grating 1: $n_{x}=1, \quad n_{y}=1 ; \quad \Lambda=\frac{1}{\sqrt{2}}=0.707 \mu \mathrm{m}, \quad \beta=45^{\circ}$,

Grating $2: \quad n_{x}=4, \quad n_{y}=3 ; \quad \Lambda=\frac{1}{\sqrt{25}}=0.2 \mu \mathrm{m}, \quad \beta=36.87^{\circ}$,

Grating $3: \quad n_{x}=3, \quad n_{y}=-4 ; \quad \Lambda=\frac{1}{\sqrt{25}}=0.2 \mu \mathrm{m}, \quad \beta=-53.13^{\circ}$.

The value of $\beta$ is in the range $\left(-90^{\circ}, 90^{\circ}\right)$, in which the positive value corresponds to clockwise rotation with respect to the $x$ axis, and the negative values correspond to counterclockwise rotation. Since the choice of $f_{s x}$ and $f_{s y}$ can be arbitrary and there are many combinations of $\left(n_{x}, n_{y}\right)$, this sampling-frequency-based SRG design scheme is very powerful and flexible.

Once $\left(f_{s x}, f_{s y}\right)$ is chosen and the sampling lattice is fixed, it is straightforward to apply RCWA analysis to SRGs. In SRG-RCWA, all grating layers in the SRG can be expanded into Fourier series of $\left(f_{s x}, f_{s y}\right)$. Similar to the standard 3D RCWA, the fields in the incident, grating, and exit regions are expanded into sampling harmonics in SRG-RCWA. With the field expressions for each region known, a linear equation system for the unknown field expansion coefficients can be derived by matching the boundary conditions at each layer interface in the SRG. To avoid numerical stability issues, we extended the enhanced transmittance matrix method ${ }^{10}$ to the $3 \mathrm{D}$ case and simultaneously solved the system of equations. The diffraction efficiency of each sampling harmonic is calculated with the same procedure as in the 3D RCWA algorithm. Since the numerical steps in SRG-RCWA are very similar to those of the standard 3D RCWA, we do not give all the tedious mathematical derivations and instead refer the interested reader to the literature for details. Here we present only the modifications to the standard 3D RCWA algorithm to incorporate the sampling frequency of SRG-RCWA.

\section{B. SRG-RCWA Modification to the Standard 3D RCWA Algorithm}

In SRG-RCWA, the arbitrary sampling frequencies $f_{s x}$ and $f_{s y}$ along the $x$ and $y$ directions are used, instead of $1 / \Lambda_{x}$ and $1 / \Lambda_{y}$ in the standard 3D RCWA. There- fore the periodic permittivity and the inverse permittivity of each grating layer in the SRG stack is expanded in a Fourier series of the sampling harmonics as

$$
\begin{aligned}
\varepsilon_{\ell}(x, y)= & \sum_{g, h} \varepsilon_{\ell, g h} \exp \left[j\left(2 \pi g f_{s x} x+2 \pi h f_{s y} y\right)\right], \\
\varepsilon_{\ell}^{-1}(x, y)= & \sum_{g, h} A_{\ell, g h} \exp \left[j\left(2 \pi g f_{s x} x+2 \pi h f_{s y} y\right)\right], \\
\varepsilon_{\ell, g h}= & \frac{1}{\Lambda_{s x}} \frac{1}{\Lambda_{s y}} \int_{0}^{\Lambda_{s x}} \int_{0}^{\Lambda_{s y}} \varepsilon_{\ell}(x, y) \\
& \times \exp \left[-j\left(2 \pi g f_{s x} x+2 \pi h f_{s y} y\right)\right] \mathrm{d} x \mathrm{~d} y, \\
A_{\ell, g h}= & \frac{1}{\Lambda_{s x}} \frac{1}{\Lambda_{s y}} \int_{0}^{\Lambda_{s x}} \int_{0}^{\Lambda_{s y}} \varepsilon_{\ell}^{-1}(x, y) \\
& \times \exp \left[-j\left(2 \pi g f_{s x} x+2 \pi h f_{s y} y\right)\right] \mathrm{d} x \mathrm{~d} y, \\
k_{x m}= & k_{0}\left[n_{1} \sin \theta \cos \varphi-m\left(\lambda_{0} f_{s x}\right)\right], \\
k_{y n}= & k_{0}\left[n_{1} \sin \theta \sin \varphi-n\left(\lambda_{0} f_{s y}\right)\right],
\end{aligned}
$$

where $\Lambda_{s x}=1 / f_{s x}$ and $\Lambda_{s y}=1 / f_{s y}$ are the sampling periods in the $x$ and $y$ directions, respectively; $m$ and $n$ are the $m$ th and $n$th sampling harmonics in the $x$ and $y$ directions, respectively; and $l$ is the grating layer index in the stack. These equations are similar to those in Ref. 8 for the standard 3D RCWA algorithm. The formation of Toeplitz permittivity and inverse-permittivity matrices from $\varepsilon_{\ell, g h}$ and $A_{\ell, g h}$ is the same in $3 \mathrm{D}$ RCWA and hence is not repeated here.

It should be pointed out that one can greatly improve the computational efficiency by using $1 \mathrm{D}$ integration to get $\varepsilon_{\ell, g h}$ and $A_{\ell, g h}$ instead of $2 \mathrm{D}$ integration in Eqs. (8) and (9):

$$
\begin{aligned}
\varepsilon_{\ell, g^{\prime}} & =\frac{1}{\Lambda} \int_{0}^{\Lambda} \varepsilon_{\ell}\left(x^{\prime}\right) \exp \left(-j \frac{2 \pi}{\Lambda} g^{\prime} x^{\prime}\right) \mathrm{d} x^{\prime}, \\
A_{\ell, g^{\prime}} & =\frac{1}{\Lambda} \int_{0}^{\Lambda} \varepsilon_{\ell}^{-1}\left(x^{\prime}\right) \exp \left(-j \frac{2 \pi}{\Lambda} g^{\prime} x^{\prime}\right) \mathrm{d} x^{\prime},
\end{aligned}
$$

where $x^{\prime}$ is the direction of the $\bar{K}$ vector of the grating. However, it is critical to put these coefficients onto the correct positions of the Toeplitz permittivity and inverse-permittivity matrices.

There are several points worth mentioning for the proposed SRG-RCWA. First, note in Eq. (1) that the grating period $\Lambda$ is inversely related to the square root of the product of the sampling numbers and the sampling frequencies. It is very important to maximize $\left(f_{s x}, f_{s y}\right)$ and to minimize $\left(n_{x}, n_{y}\right)$ when designing SRGs in order to improve numerical efficiency and accuracy. As in standard 3D RCWA, only a finite number of sampling harmonics $N_{\max }$ can be kept in 
the SRG-RCWA calculation. For a given $N_{\max }$, the number of effective harmonics for a particular grating is given by int $\left[N_{\max } / n_{x}\right]$ for the $x$ direction, where the int [ ] function means the integer part of the operand. It is apparent that the results will be more accurate for smaller $n_{x}$ because more harmonics are kept in the calculation.

Second, for a given SRG not designed in accordance with the above-proposed sampling frequency concept, it may be that no $\left(f_{s x}, f_{s y}\right)$ pair can match the parameters of all of the gratings in the stack. In such cases, some compromise must be made to apply SRGRCWA. Usually a slightly different grating period and/or its orientation angle must be used instead of their actual parameters to enforce all the diffraction orders generated by the grating laid on the sampling frequency lattice. However, in many cases accurate simulation results can still be achieved as demonstrated in Section 3.

The proposed sampling frequency and sampling lattice-based SRG design principle is particularly suitable to designing SRGs as beam splitters for producing rectangular spot arrays with a small number of spots, for instance, $3 \times 3$ or $4 \times 4$ arrays. In particular, SRG-RCWA is useful when the angular separation between spots is so large that it is inefficient, if not impossible, to design such beam splitters in the paraxial diffraction domain because of the required small grating periods. ${ }^{11}$ However, such beam splitters can be efficiently realized by SRGs and can be rigorously designed with the SRG-RCWA method. For example, a $3 \times 3$ spot array beam splitter can be realized by a SRG with three gratings rotated at $0^{\circ}$, $45^{\circ}$, and $90^{\circ}$ with respect to each other. All of the gratings will need to generate only the $-1,0$, and +1 orders as propagating orders. The $0^{\circ}$ and $90^{\circ}$ oriented gratings are identical except for their orientations, and the grating period of the middle layer $45^{\circ}$ grating is $\sqrt{2} / 2$ of those of the other two. Such a SRG creates a $3 \times 3$ lattice in $k$ space. Since all of the incident power can be diffracted only to the desired orders, the efficiency of such a beam splitter is expected to be high. Furthermore, because of the symmetric orientations of the gratings, such a SRG-based beam splitter is insensitive to the polarization of the incident light, which is advantageous in many applications.

\section{Validation of the SRG-RCWA Algorithm}

In this subsection, we use a three-layer sandwich SRG to validate our SRG-RCWA algorithm. The sandwich grating stack has two grating layers and a homogeneous layer in between. The two grating layers are two identical 1D surface-relief gratings with orthogonal grating vectors, as shown in Fig. 3. The gratings are $1 \mu \mathrm{m}$ in period with a $50 \%$ fill factor. The thickness of the grating layers is $1 \mu \mathrm{m}$. The refractive indices of the ridge region and the groove region are 2.5 and 1.5 , respectively. The index of refraction of the homogeneous layer is 2.5 , and the thickness is $2 \mu \mathrm{m}$. The indices of refraction of the incident and exit regions

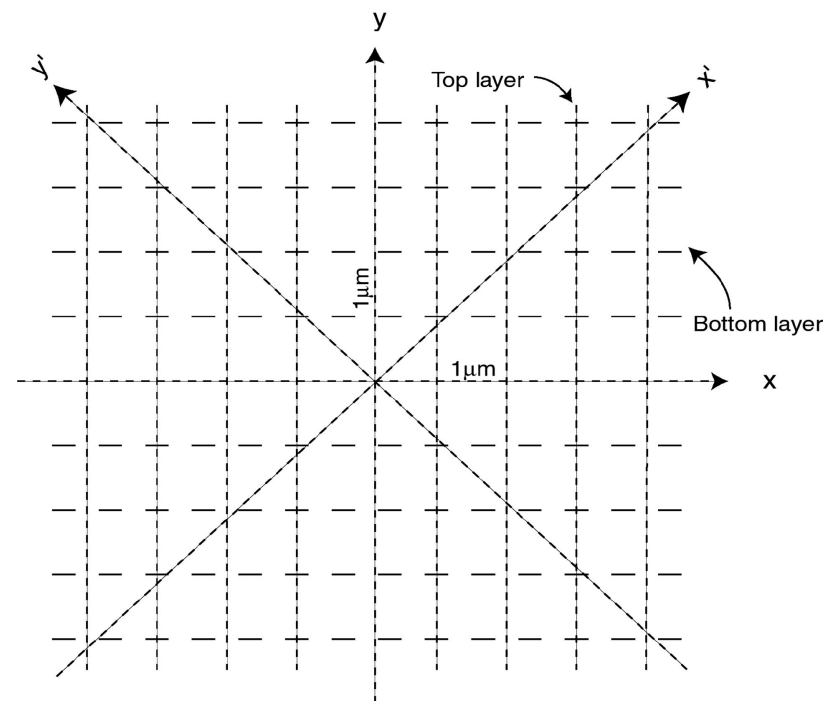

Fig. 3. Coordinate system of the sandwich grating stack.

are 1.0 and 2.5, respectively. The wavelength of the incident light is $5 \mu \mathrm{m}$.

Since the two grating vectors are perpendicular to each other, the standard 3D RCWA can be applied to the stack. In addition, the stack can also be analyzed with SRG-RCWA in two coordinate systems. In one system the $x$ and $y$ axes are parallel to the two grating vectors ( $x-y$ system in Fig. 3$)$ and the other is rotated $45^{\circ}$ relative to the $x-y$ system $\left(x^{\prime}-y^{\prime}\right.$ system in Fig. 3$)$. The sampling frequencies are $f_{s x}=f_{s y}=1 \mu \mathrm{m}^{-1}$ in the $x-y$ system and $f_{s x}{ }^{\prime}=f_{s y}{ }^{\prime}=\sqrt{2} / 2 \mu \mathrm{m}^{-1}$ in the $x^{\prime}-y^{\prime}$ system. TE polarization with respect to the $x-y$ coordinate system is studied with these three methods. The diffraction efficiency of the transmitted zero order as a function of the number of orders retained in the calculation is compared in Fig. 4. The results of standard 3D RCWA and SRG-RCWA in the $x-y$ coordinate system match very well, while SRG-RCWA

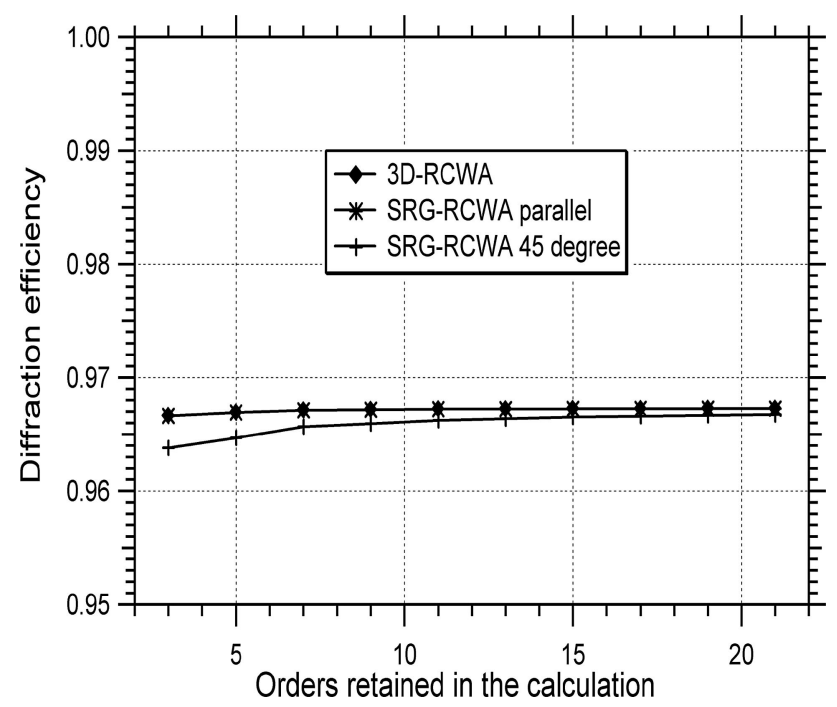

Fig. 4. Comparison of three algorithms on the sandwich grating stack. 
in the $x^{\prime}-y^{\prime}$ system converges somewhat more slowly and therefore requires more orders to be retained to obtain a given accuracy. This is not surprising since the convergence of the RCWA algorithm is highly dependent on the profile of the grating. ${ }^{12}$ However, the difference in diffraction efficiency between standard 3D RCWA and SRG-RCWA in the $x^{\prime}-y^{\prime}$ system is less than $0.05 \%$ if $441(21 \times 21)$ sampling harmonics are retained in both calculations, which is accurate enough for most applications.

\section{SRG-RCWA Characterization of Two Circular Polarization Filters}

In this section, we apply SRG-RCWA to characterize two fabricated stacked rotated gratings as circular polarization filters as discussed in Ref. 5. As mentioned in Section 1, each filter consists of a wire-grid polarizer and a subwavelength dielectric grating designed as a quarter-wave plate and rotated $45^{\circ}$ with respect to the wire-grid polarizer. Scanning electron microscopy (SEM) images of the two fabricated polarizer SRG devices are shown in Figs. 7 and 10 in Ref. 5, respectively. The detailed structural layer parameters for each device are listed in Table 1. Note that a commercial broadband antireflection coating for the 3-5 $\mu \mathrm{m}$ wavelength range was applied to the backs of the sample wafers to suppress Fresnel reflections occurring at that interface, which are not

Table 1. Detail Structural Parameters of Two SRG Sample Devices as Circular Polarization Detectors

\begin{tabular}{|c|c|c|c|}
\hline & & Stack 1 & Stack 2 \\
\hline \multicolumn{2}{|c|}{ Incident Media } & $\mathrm{Si}$ & $\mathrm{Si}$ \\
\hline \multirow{6}{*}{$\begin{array}{l}\text { Quarter-wave plate } \\
\text { Sublayer } 1\end{array}$} & & & \\
\hline & Ridge material & $\mathrm{Si}$ & $\mathrm{Si}$ \\
\hline & Groove material & Su-8 & $\mathrm{Su}-8$ \\
\hline & $\begin{array}{l}\text { Grating period } \\
(\mu \mathrm{m})\end{array}$ & 1.0 & 1.0 \\
\hline & Fill factor & $62.2 \%$ & $61.3 \%$ \\
\hline & Thickness $(\mu \mathrm{m})$ & 0.72 & 0.5 \\
\hline \multirow[t]{5}{*}{ Sublayer 2} & Ridge material & $\mathrm{Si}$ & $\mathrm{Si}$ \\
\hline & Groove material & Su-8 & $\mathrm{Su}-8$ \\
\hline & $\begin{array}{l}\text { Grating period } \\
(\mu \mathrm{m})\end{array}$ & 1.0 & 1.0 \\
\hline & Fill factor & $53.4 \%$ & $54.2 \%$ \\
\hline & Thickness $(\mu \mathrm{m})$ & 1.21 & 1.53 \\
\hline \multirow{2}{*}{$\begin{array}{l}\text { SU-8 homogeneous } \\
\text { layer }\end{array}$} & Refractive index & Varying & Varying \\
\hline & Thickness $(\mu \mathrm{m})$ & 1.26 & 1.47 \\
\hline \multirow{2}{*}{$\begin{array}{l}\mathrm{SiO}_{2} \text { homogeneous } \\
\text { layer }\end{array}$} & Refractive index & Varying & - \\
\hline & Thickness $(\mu \mathrm{m})$ & 0.1 & - \\
\hline \multirow[t]{5}{*}{ Polarizer } & Ridge material & Molybdenum & Molybdenum \\
\hline & Groove material & Air & Air \\
\hline & $\begin{array}{l}\text { Grating period } \\
(\mu \mathrm{m})\end{array}$ & 0.5 & 1.0 \\
\hline & Fill factor & $33 \%$ & $41 \%$ \\
\hline & Thickness $(\mu \mathrm{m})$ & 0.16 & 0.16 \\
\hline \multirow[t]{6}{*}{ Cr layer } & Ridge material & $\mathrm{Cr}$ & $\mathrm{Cr}$ \\
\hline & Groove material & Air & Air \\
\hline & $\begin{array}{l}\text { Grating period } \\
(\mu \mathrm{m})\end{array}$ & 0.5 & 1.0 \\
\hline & Fill factor & $70 \%$ & $58 \%$ \\
\hline & Thickness $(\mu \mathrm{m})$ & 0.025 & 0.27 \\
\hline & Exit media & Air & Air \\
\hline
\end{tabular}

shown in the SEM images. The wire-grid polarizers in these two devices are different. Stack 1 has a grating period of $0.5 \mu \mathrm{m}$, while Stack 2 has a grating period of $1.0 \mu \mathrm{m}$. Also an additional Cr grating layer is evaporated on top of the molybdenum layer to protect the polarizer. In Stack 1 there is an extra $\mathrm{SiO}_{2}$ homogeneous layer, which serves as an etch stop for reactive ion etching in the fabrication process.

The performance of these SRG devices as circular polarization filters is experimentally characterized by measuring the transmissions of incident left- and right-circular polarized (LCP and RCP, respectively) illumination. A Fourier transform infrared (FTIR) spectrometer (FTS-60A from BioRad, Digilab Division, Cambridge, Massachusetts) and an FTIR spectropolarimeter were used to obtain measurements across the desired wavelength range of 3.5-5 $\mu \mathrm{m}$. The detailed experiment setup is shown in Fig. 8 of Ref. 5. The experimental transmission curves for both sample stacks are shown here in Figs. 5(a) and 6(a), respectively. A key performance parameter, the circular extinction ratio (CER), can be defined as

$$
\mathrm{CER} \equiv T_{R C P} / T_{L C P},
$$

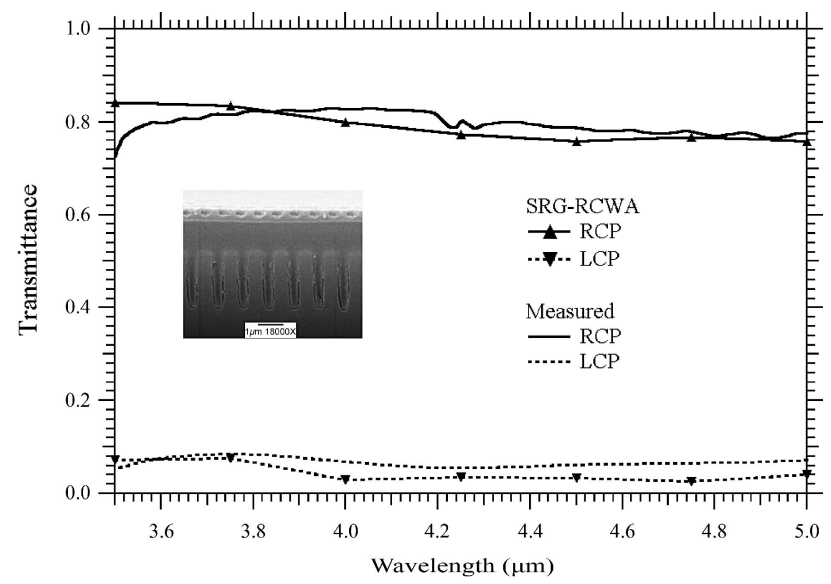

(a)

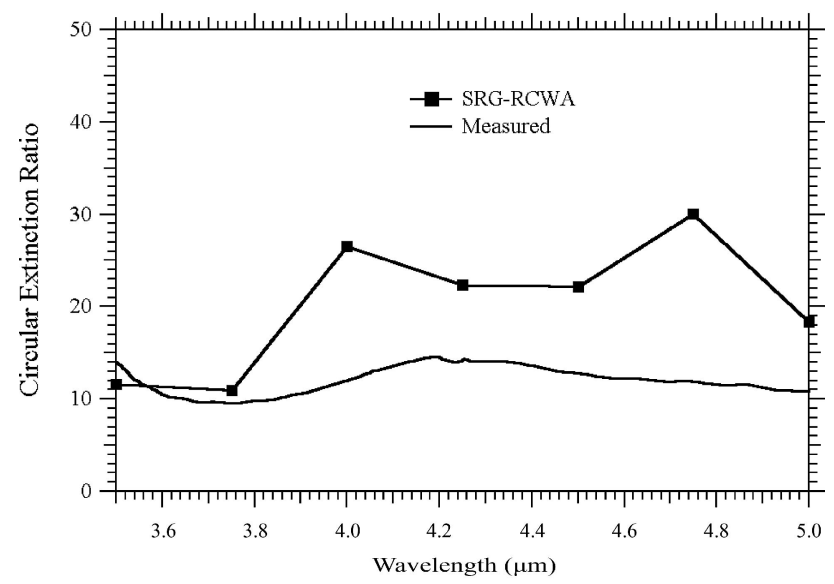

(b)

Fig. 5. (a) Transmittance and (b) extinction ratio of SRG sample Stack 1. 


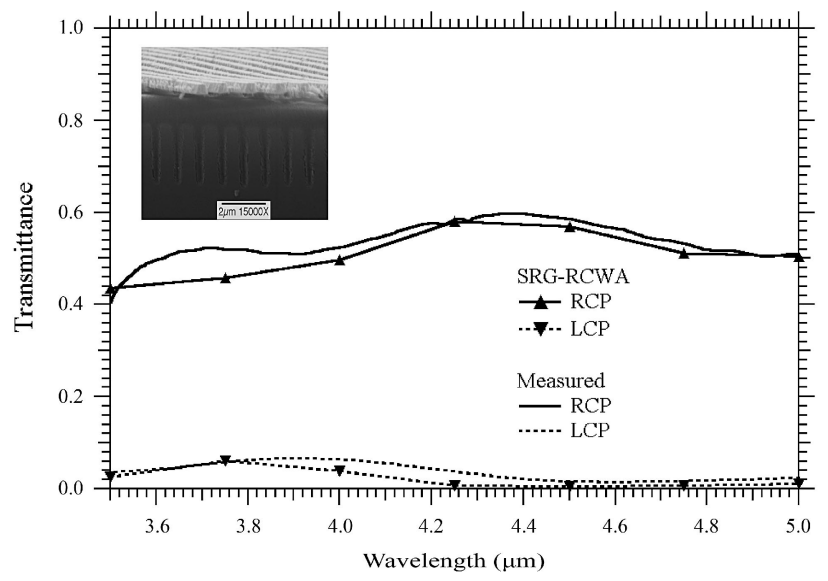

(a)

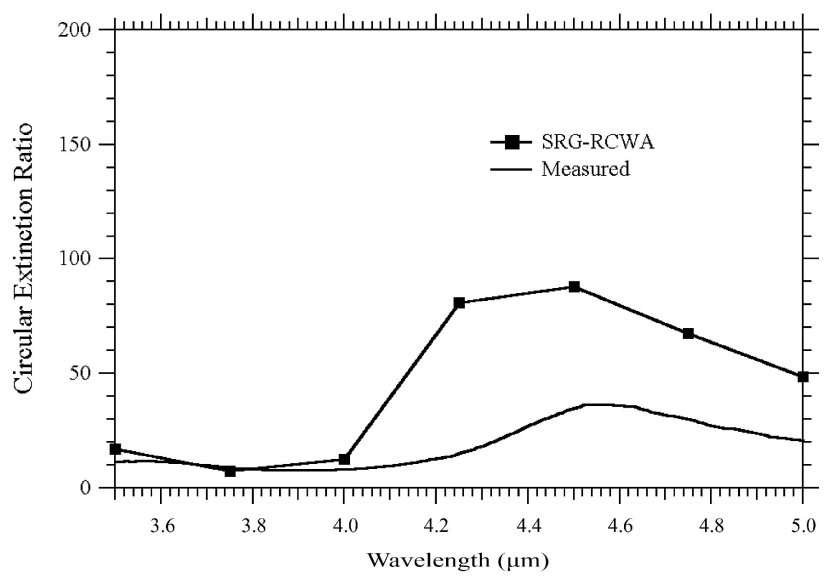

(b)

Fig. 6. (a) Transmittance and (b) extinction ratio of SRG sample Stack 2 .

in which $T_{R C P}$ and $T_{L C P}$ are the transmissions of RCP and LCP light, respectively. The measured CER curves for both stacks are presented in Figs. 5(b) and $6(\mathrm{~b})$, respectively.

To apply the SRG-RCWA algorithm for the rigorous analysis of these two SRG circular polarization filters, one must first determine the sampling frequencies and sampling numbers for both the quarter-wave plate and the wire-grid polarizer. Unfortunately, the wire-grid polarizer and the quarter-wave plate were independently designed with a standard 1D RCWA algorithm and did not follow the sampling frequency concept. For both sample devices, no pair of $\left(f_{s x}, f_{s y}\right)$ can exactly match its structural configurations (grating periods and angular orientations). Therefore some compromise must be made to apply SRG-RCWA. The final selected values for these parameters are listed in Table 2. Note for both devices, the $x$ axis of the coordinate system is along the grating vector of the wire-grid polarizers. The sampling frequencies and sampling numbers exactly match the configurations of the quarter-wave plates while approximations are made for the grating periods of the polarizers. According to Eq. (1) it is straightforward to calcu-
Table 2. SRG-RCWA Sampling Frequencies and Sampling Numbers for the Simulation of Two SRG Sample Devices

\begin{tabular}{lccc}
\hline & & Device 1 & Device 2 \\
\hline Sampling & $f_{s x}$ & $\sqrt{2} / 2$ & $\sqrt{2} / 4$ \\
frequency & $f_{s y}$ & $\sqrt{2} / 2$ & $\sqrt{2} / 4$ \\
Quarter-wave & $n_{x}$ & 1 & 2 \\
plate & $n_{y}$ & -1 & -2 \\
& Grating period $(\mu \mathrm{m})$ & 1.0 & 1.0 \\
Polarizer & $n_{x}$ & 3 & 3 \\
& $n_{y}$ & 0 & 0 \\
& Grating period $(\mu \mathrm{m})$ & 0.4714 & 1.0607 \\
\hline
\end{tabular}

late the actual grating periods used in the SRGRCWA analysis, which are 0.4714 and $1.0607 \mu \mathrm{m}$, respectively. These periods deviate slightly from the designed values of 0.5 and $1.0 \mu \mathrm{m}$.

Another issue in the SRG-RCWA analysis is the imperfect circular polarization of the incident beam caused by the imperfect components used in the measurement to generate CP light. The incident beam is actually elliptically polarized. However, the polarization state information can be determined by the Jones Matrix method ${ }^{13}$ combined with the measured performance of the components used. Table 3 shows the detailed input elliptical polarization parameters used in SRG-RCWA simulation, in which $a$ and $b$ are the major and minor half-axis of the elliptical polarization, and $\Psi$ is the angle between the major axis and the $x$ axis. In the SRG-RCWA simulations, the quarter-wave plate is divided into two sublayers to approximate the nonrectangular etched grating profile, as shown in Table 1. Overall, six different layers are used to simulate Stack 1, and five layers are used in the Stack 2 simulation.

The SRG-RCWA simulation results for these two devices are illustrated in Figs. 5 and 6, along with the measurement results to facilitate direct comparison. For both devices, the SRG-RCWA results match the corresponding measurements very well for RCP light. However, the LCP measured values are higher than the simulation results, which causes the apparent disagreement between simulation and measurements for the CER because CER is very sensitive to LCP transmission. Two factors may account for this disagreement. From the point of view of numerical simulation,

Table 3. Input Elliptical Polarization Parameters to SRG-RCWA Simulation

\begin{tabular}{lccc}
\hline $\begin{array}{c}\lambda \\
(\mu \mathrm{m})\end{array}$ & $b / a$ & $\begin{array}{c}\psi_{R C P} \\
\left({ }^{\circ}\right)\end{array}$ & $\begin{array}{c}\psi_{L C P} \\
\left({ }^{\circ}\right)\end{array}$ \\
\hline 3.5 & 0.75 & 94.38 & 85.62 \\
3.75 & 0.75 & 94.08 & 85.92 \\
4 & 0.79 & 90.40 & 89.60 \\
4.25 & 0.85 & 87.21 & 92.79 \\
4.5 & 0.92 & 86.87 & 93.13 \\
4.75 & 0.98 & 79.93 & 100.07 \\
5 & 0.97 & 30.50 & 149.50 \\
\hline
\end{tabular}


the selected sampling frequencies and sampling numbers resulted in somewhat different grating periods for both polarizers. In addition, only $15 \times 15$ harmonic orders are kept in the simulations. Also, considering the experimental measurement details, the broadband antireflection coating at the silicon-air interface achieves transmission of $95 \%$ to $98.5 \%$ across the whole wavelength range of interest. The residual reflection may cause multiple reflections in the Si substrate. The LCP mode is affected more than the RCP because the CP filters are designed to block LCP and therefore more light undergoes multiple reflections. On the other hand, the wire-grid polarizer has some defects because of fabrication errors. Some small regions are missing wire-grid gratings and are therefore transparent to any incident polarization. Despite these imperfections, the SRG-RCWA simulation results provide a reasonable match to the measured transmission data.

\section{Conclusion}

We have developed a sampling frequency concept to systematically design multilayer stacked rotated gratings. With an arbitrarily chosen sampling rectangular lattice, all the $1 \mathrm{D}$ gratings in the stack can be designed so that the resultant diffraction orders are located only on the lattice grids. To rigorously design and analyze the SRGs, we also extended the standard 3D RCWA algorithm with the concept of sampling frequency and sampling harmonics. With the latest SRG-RCWA algorithm, two fabricated SRGs as circular polarization filters have been successfully characterized. Good agreement between the experimentally measured transmission and the SRG-RCWA simulation has been achieved.

\section{References}

1. E. Loewen and E. Popov, Diffraction Gratings and Applications (Marcel Dekker, 1997).

2. E. G. Johnson, "Design and analysis of single and cascaded diffractive optical elements," Ph.D. dissertation (The University of Alabama in Huntsville, 1996).

3. D. Chambers, "Stratified volume diffractive optical elements," Ph.D. dissertation (The University of Alabama in Huntsville, 2000).

4. D. Chambers, G. P. Nordin, and S. Kim, "Fabrication and analysis of a three-layer stratified volume diffractive optical element high-efficiency grating," Opt. Express 11, 27-38 (2003).

5. R. B. Hwang and S. T. Peng, "Performance evaluation of a bigrating as a beam splitter," Appl. Opt. 36, 2011-2018 (1997).

6. S. Lin and J. G. Fleming, "Creation of a 3D silicon photonic crystal," Opt. Photon. News 9(12), 35-37 (1998).

7. P. C. Deguzman and G. Nordin, "Stacked subwavelength gratings as circular polarization filters," Appl. Opt. 40, 5731-5737 (2001).

8. M. G. Moharam and T. K. Gaylord, "Three-dimensional vector coupled-wave analysis of planar-grating diffraction," J. Opt. Soc. Am. 73, 1105-1112 (1983).

9. R. Brauer and O. Bryngdahl, "Electromagnetic diffraction analysis of two-dimensional gratings," Opt. Commun. 100, 1-5 (1993).

10. M. G. Moharam, D. A. Pommet, and E. B. Grann, "Stable implementation of the rigorous coupled-wave analysis for surface relief gratings: enhanced transmittance matrix approach," J. Opt. Soc. Am. A 12, 1077-1086 (1995).

11. J. Turunen and F. Wyrowski, Diffractive Optics for Industrial and Commercial Applications (Akademie Verlag, 1997).

12. P. Lalanne, "Improved formulation of the coupled-wave method for two-dimensional gratings," J. Opt. Soc. Am. A 14, 1592-1598 (1997).

13. P. C. Deguzman, "Stacked subwavelength gratings for imaging polarimetry," Ph.D. dissertation (The University of Alabama in Huntsville, 2000). 